\title{
Superior efficacy of cotreatment with BET protein inhibitor and BCL2 or MCL1 inhibitor against AML blast progenitor cells
}

\author{
Warren Fiskus ${ }^{1}$, Tianyu Cai ${ }^{1}$, Courtney D. DiNardo (1)', Steven M. Kornblau', Gautam Borthakur', Tapan M. Kadia', \\ Naveen Pemmaraju', Prithviraj Bose', Lucia Masarova', Kimal Rajapakshe², Dimuthu Perera ${ }^{2}$, Cristian Coarfa²,

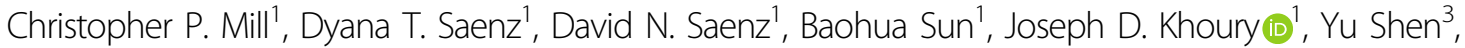 \\ Marina Konopleva ${ }^{1}$ and Kapil N. Bhalla ${ }^{1}$
}

\begin{abstract}
First-generation bromodomain extra-terminal protein (BETP) inhibitors (BETi) (e.g., OTX015) that disrupt binding of BETP BRD4 to chromatin transcriptionally attenuate AML-relevant progrowth and prosurvival oncoproteins. BETi treatment induces apoptosis of AML BPCS, reduces in vivo AML burden and induces clinical remissions in a minority of AML patients. Clinical efficacy of more potent BETis, e.g., ABBV-075 (AbbVie, Inc.), is being evaluated. Venetoclax and A1210477 bind and inhibit the antiapoptotic activity of BCL2 and MCL1, respectively, lowering the threshold for apoptosis. BETi treatment is shown here to perturb accessible chromatin and activity of enhancers/promoters, attenuating MYC, CDK6, MCL1 and BCL2, while inducing BIM, HEXIM1, CDKN1A expressions and apoptosis of AML cells. Treatment with venetoclax increased MCL1 protein levels, but cotreatment with ABBV-075 reduced MCL1 and BCl-xL levels. ABBV-075 cotreatment synergistically induced apoptosis with venetoclax or A-1210477 in patient-derived, CD34 + AML cells. Compared to treatment with either agent alone, cotreatment with ABBV-075 and venetoclax was significantly more effective in reducing AML cell-burden and improving survival, without inducing toxicity, in AMLengrafted immune-depleted mice. These findings highlight the basis of superior activity and support interrogation of clinical efficacy and safety of cotreatment with BETi and BCL2 or MCL1 inhibitor in AML.
\end{abstract}

\section{Introduction}

The bromodomain extra-terminal (BET) protein (BETP) BRD4 interacts with transcription factors as well as cofactors, including mediator protein complex, lysine methyltransferase NSD3, arginine demethylase JMJD6, and pTEFb (a heterodimer of CDK9 and cyclin T), to regulate RNA pol II (RNAP2)-mediated transcript elongation ${ }^{1-4}$. BRD4 promotes pTEFb-mediated phosphorylation of serine 2 in the heptad repeats within the CTD of RNAP2, as well as of the

\footnotetext{
Correspondence: Kapil N. Bhalla (kbhalla@mdanderson.org)

${ }^{1}$ The University of Texas MD Anderson Cancer Center, Houston, TX 77030, USA ${ }^{2}$ Department of Molecular and Cellular Biology, Baylor College of Medicine, Houston, TX 77030, USA

Full list of author information is available at the end of the article. These authors contributed equally: Warren Fiskus, Tianyu Cai, Marina Konopleva, Kapil N. Bhalla
}

negative transcription elongation factors, NELF and Sept5, which induces promoter-proximal pause release of RNAP2 and RNA transcript elongation ${ }^{4-6}$. This has been shown to occur at the enhancers and promoters of oncogenes that promote growth and survival of cancer cells, including acute myeloid leukemia (AML) stem-progenitor cells ${ }^{2,6-9}$. Consistent with this, knockdown of BRD4 by RNAi, or disruption of its binding to acetylated chromatin by BET inhibitors (BETi) leads to lethality in AML blast progenitor cells (BPCs), associated with down regulation of AMLrelevant progrowth and prosurvival oncogenes ${ }^{1,2,10-13}$. BETis, including JQ1 and OTX015, have been documented to reduce AML burden and improve survival of mice engrafted with human AML BPCs ${ }^{11-13}$. Whereas treatment with BETi was shown to induce clinical responses in AML, 
refractoriness to BETi therapy and resistance with disease progression is uniformly observed ${ }^{14-16}$. This has prompted the development and testing of more potent and effective BETis, e.g., ABBV-075 ${ }^{16-20}$. Since BETi treatment attenuated expressions of several BCL2 family of antiapoptotic proteins ${ }^{11-13,21}$, to further lower the threshold for apoptosis and enhance clinical anti-AML efficacy of BETi, a logical approach would be to concomitantly target and inhibit activity of the antiapoptotic proteins.

BCL2, Bcl-xL, and MCL1 are members of multi-BCL-2 homology (BH) domain (BH1-BH4) containing family of antiapoptotic proteins ${ }^{22,23}$. They bind proapoptotic BCL2 family members BAX and BAK (containing BH1, BH2, and $\mathrm{BH} 3$ ) and $\mathrm{BH} 3$ domain-only proapoptotic activator proteins, to inhibit intrinsic mitochondria-induced pathway of apoptosis ${ }^{22-24}$. The first, highly selective BCL2 inhibitor venetoclax (ABT-199) binds specifically to BCL2 and displaces $\mathrm{BH} 3$ domain-only proteins to trigger BAX/ BAK-mediated mitochondria-induced apoptosis of cancer, including AML cells ${ }^{25,26}$. Venetoclax treatment alone showed anti-AML in vivo efficacy in the mouse xenograft models ${ }^{26,27}$. Although effective in inducing clinical remissions in AML, innate or acquired resistance to venetoclax alone is commonly observed ${ }^{28}$. The best predictor of sustained response to venetoclax is the lack of readily accessible resistance mechanisms provided by Bcl$\mathrm{xL}$ and $\mathrm{MCL}^{28}$. In venetoclax-resistant cells, increased MCL1 and/or Bcl-xL levels was observed ${ }^{29}$. Preclinically, dual targeting of BCL2 and MCL1, but not either alone, was also shown to prolong survival of AML or lymphoma bearing mice ${ }^{30,31}$. Combining venetoclax with other antiAML drugs such as cytarabine or DNA hypomethylating agent has yielded higher remission rates ${ }^{32,33}$. However, a full assessment of their clinical efficacy has not been conducted. In present studies we determined the effects of the BETi on cis-regulatory DNA elements and on mRNA and protein expressions of AML-relevant oncoproteins, including MYC, BCL2, Bcl-xL, MCL1, and CDK6. We also determined whether the BETi ABBV-075-induced perturbations in protein levels of these oncoproteins are associated with synergistic in vitro activity of ABBV-075 and venetoclax or the MCL1 inhibitor A-1210477. Finally, we also determined whether combination of ABBV-075 and venetoclax exerted superior in vivo efficacy against mouse xenograft models of AML BPCs.

\section{Materials and methods \\ Reagents and antibodies}

In vivo grade ABBV-075 and ABT-199 for mouse xenograft experiments were kindly provided by AbbVie, Inc. In vitro grade A-1210477 (Catalog No. S7790), ABBV-075 (Catalog No. S8400) and ABT-199 (Catalog No. S8048) were purchased from Selleck Chemicals (Houston, TX) and utilized for in vitro experiments. All compounds were prepared as $10 \mathrm{mM}$ stocks in $100 \%$ dimethyl sulfoxide (DMSO) and frozen at $-80{ }^{\circ} \mathrm{C}$ in $5-10 \mu \mathrm{L}$ aliquots to allow for single use, thus avoiding multiple freeze-thaw cycles that could result in compound decomposition and loss of activity. Anti-BRD4 (RRID: AB_2620184) antibody was obtained from Bethyl Labs (Montgomery, TX). Anti-c-Myc (RRID: AB_1903938), anti-HEXIM1 (Cat \#12604), anti-p21 (RRID: AB_823586), anti-p-Histone H2AX (Ser139) (RRID: AB_2118010), anti-Bcl-xL (RRID: AB_10695729), anti-BAX (RRID: AB_2744530), anti-BAK (RRID:AB_2290287), antiCleaved PARP (RRID:AB_331426), anti-BIM (RRID: AB_1030947) and anti-MCL1 (RRID:AB_2281980) antibodies were obtained from Cell Signaling Technologies (Beverly, MA). Anti-CDK6 (RRID: AB_10610066), antiBcl2 (RRID: AB_626733), Alexa488-conjugated anti-BAX (6A7) (RRID:AB_626728) and anti- $\beta$-Actin (RRID: AB_626630) antibodies were obtained from Santa Cruz Biotechnologies (Santa Cruz, CA). Anti-BAK(NT) (RRID: AB_310159) antibody was obtained from Millipore Sigma (Burlington, MA).

\section{Cell lines and cell culture}

Human AML cell lines MOLM13 (RRID:CVCL_2119), SET2 (RRID:CVCL_2187), SKM1 (RRID:CVCL_0098) and OCI-AML5 (RRID:CVCL_1620) were obtained from the DSMZ (Braunschweig, Germany). MV4-11 cells were obtained from the ATCC (Manassas, VA). All experiments with cell lines were performed within 6 months after thawing or obtaining from ATCC or DSMZ. The cell lines were also authenticated in the Characterized Cell Line Core Facility at M.D. Anderson Cancer Center, Houston TX. SKM1 and MOLM13 cells were cultured in RPMI media with $20 \%$ fetal bovine serum (FBS) and $1 \%$ penicillin/streptomycin and $1 \%$ non-essential amino acids. OCI-AML 5 cells were cultured in MEM alpha media with $20 \%$ FBS and $1 \%$ penicillin/streptomycin and $1 \%$ nonessential amino acids. OCI-AML5 cultures were also supplemented with $10 \mathrm{ng} / \mathrm{mL}$ of GM-CSF (PeproTech, Rocky Hill, NJ). MV4-11 cells were cultured in ATCCformulated Iscove's Modified Dulbecco's Medium (IMDM) with 20\% FBS 1\% penicillin/streptomycin and 1\% non-essential amino acids. Luciferase-expressing MOLM13 cells were created by transducing cells with Luc-ZSGreen(GFP). pHIV-Luc-ZsGreen was a gift from Bryan Welm (Addgene plasmid \#39196). Logarithmically growing, mycoplasma-negative cells were utilized for all experiments. Following drug treatments, cells were washed free of the drug(s) prior to the performance of the studies described.

\section{Primary AML blast cells}

Patient-derived AML cells samples were obtained with informed consent as part of a clinical protocol approved 
by the Institutional Review Board of The University of Texas, M.D. Anderson Cancer Center. Normal hematopoietic progenitor cells (HPCs) were obtained from delinked, de-identified cord blood samples. Mononuclear cells were purified by Ficoll Hypaque density centrifugation. Mononuclear cells were washed with complete RPMI media containing 20\% FBS. CD34+ AML BPCs and normal HPCs were purified by immunomagnetic beads conjugated with anti-CD34 antibody (StemCell Technologies, Vancouver, British Columbia) prior to utilization in the cell viability assays and immunoblot analyses.

RNA isolation and quantitative-polymerase chain reaction

Following the designated treatments with ABBV-075, total RNA was isolated from AML cells utilizing a PureLink RNA Mini kit from Ambion, Inc. (Austin, TX; RRID: SCR_008406) and reverse transcribed. Quantitative realtime PCR analysis for the expression of $M Y C, B C L-2$, BCL2L1 (Bcl-xL), CDK6, HEXIM1, and CDKN1A ( $p 21)$ was performed on cDNA using TaqMan probes from Applied Biosystems (Foster City, CA; RRID: SCR_005039). Relative mRNA expression was normalized to the expression of Glyceraldehyde 3-phosphate dehydrogenase $(G A P D H)$ and compared to the untreated cells.

\section{SDS-PAGE and immunoblot analyses}

Seventy five micrograms of total cell lysate were used for SDS-PAGE. Western blot analyses were performed on total cell lysates using specific antisera or monoclonal antibodies. Blots were washed with $1 \times$ PBST, then incubated in IRDye 680RD goat anti-mouse (RRID:AB_10956588) or IRDye 800CW goat anti-rabbit (RRID:AB_621843) secondary antibodies (LI-COR, Lincoln, NE) for $1 \mathrm{~h}$, washed three times in $1 \times$ Phosphate Buffered Saline with Tween ${ }^{\circledR} 20$ (PBST) and scanned with an Odyssey CLX Infrared Imaging System utilizing Image Studio 5.0 Software (RRID: SCR_015795) (LI-COR, Lincoln, NE). The expression levels of $\beta$-Actin in the cell lysates were used as the loading control for the western blots. Immunoblot analyses were performed at least twice. Representative immunoblot images were subjected to densitometry analysis.

\section{Assessment of apoptosis by annexin $\mathbf{V}$ staining}

Untreated or drug-treated cells were stained with Annexin V-FITC (Pharmingen, San Diego, CA) and TOPRO-3 iodide (Life Technologies, Carlsbad, CA) and the percentages of apoptotic cells were determined by flow cytometry. To analyze in vitro synergism between ABBV075 and ABT-199 or A-1210477 or synergy between A1210477 and ABT-199, cells were treated with in vitro grade single agents and combinations for $48 \mathrm{~h}$ and the percentages of annexin V-positive, apoptotic cells were determined by flow cytometry. The combination index
(CI) for each drug combination was calculated by median dose effect and isobologram analyses (assuming mutual exclusivity) utilizing the commercially available software Compusyn. CI values of less than 1.0 represent a synergistic interaction of the two drugs in the combination. The CI values were input into GraphPad V7.0 to create Box and Whisker plots of the range of the CI values for each cell line and drug combination.

\section{Assessment of percentage nonviable cells}

Following designated single agent or combination treatments, primary, patient-derived (PD)-AML cells were stained with trypan blue (Sigma, St. Louis, MO). The numbers of nonviable cells were determined by counting the cells that exhibited trypan blue uptake in a hemocytometer, and were reported as a percentage of the untreated control cells. Alternatively, cells were washed with $1 \times$ PBS, stained with TO-PRO-3 iodide and analyzed by flow cytometry on an Accuri CFlow6 flow cytometer.

\section{AML xenograft models}

All animal studies were performed under a protocol approved by the IACUC at M.D. Anderson Cancer Center, an AAALAC-accredited institution. To determine the in vivo effects of ABT-199 and/or ABBV-075 on leukemia progression and engraftment, 2 million MOLM13/GFPLuc cells were injected with a 26-gauge needle into the lateral tail vein of 4-6-week-old female NOD-scid IL2Rgamma $^{\text {null }}$ (NSG, Stock number 005557 (RRID: IMSR_JAX:005557); The Jackson Laboratory, Bar Harbor, ME) mice $(n=7)$ which had received a preconditioning dose of radiation ( $2.5 \mathrm{~Gy}) 24 \mathrm{~h}$ prior to injection of cells. All the mice were monitored for 4 days and imaged to document engraftment. Mice were randomly assigned to treatment cohorts. Following this, mice were treated daily with vehicle (10\% ethanol, 30\% PEG400, 60\% Phosal 50), $50 \mathrm{mg} / \mathrm{kg}$ of ABT-199 (by oral gavage, daily $\times 5$ days per week), $1 \mathrm{mg} / \mathrm{kg}$ of ABBV-075 (by oral gavage, daily $\times$ 5 days per week) or ABBV-075 + ABT-199 for 2 weeks. Mice were injected with $75 \mathrm{mg} / \mathrm{kg}$ of D-Luciferin and imaged once per week by Xenogen camera to monitor disease status and treatment efficacy. Mice that became moribund or experienced hind limb paralysis were euthanized according to the approved IACUC protocol. Investigators were not blinded to the experimental conditions; however, veterinarians and veterinary staff assisting in determining when euthanasia was required were blinded to the experimental conditions of the study. The survival of the mice is represented by a Kaplan-Meier plot. The variance between cohorts was similar. To determine the antileukemia effects of ABBV-075 and/or ABT-199 against human AML patient-derived xenograft (PDX) models, NSG mice ( $n=8$ per cohort) were injected 
with patient-derived AML blast cells following a preconditioning dose of radiation. Mice were monitored for engraftment by flow cytometry. Upon engraftment, mice were treated with ABBV-075 and/or ABT-199 for 22 days. At the end of the treatment period, mice were humanely euthanized and the \% of engraftment was documented in the mice.

\section{Statistical analysis}

Significant differences between values obtained in a population of AML cells treated with different experimental conditions were determined using the Student's $t$ test. For the in vivo mouse models, a two-tailed $t$ test or a Mantel-Cox Rank sum test was utilized for group comparisons. $p$ values of $<0.05$ were assigned significance.

\section{Results}

\section{BETi-mediated effects on the gene-regulatory elements} and gene-expressions in AML cells

We first determined the effects of BETi treatment on the open and accessible chromatin, at enhancers and promoters, for transcriptional complexes in AML cells, utilizing ATAC-Seq analysis. Figure 1a, panel a demonstrates large numbers of lost and gained peaks in the chromatin of the AML SET2 cells treated with the BETi OTX015 over untreated SET2 cells. This indicated that BETi treatment markedly affected the accessibility of chromatin to transcriptional complexes. Figure $1 \mathrm{~b}$ shows log2-fold-change in the ATAC-Seq peaks mapped to transcription start sites $\pm 10 \mathrm{~kb}$ in the DNA of the indicated genes. Notably, BETi treatment increased chromatin accessibility at the promoters and other cis-regulatory regions of genes, including $M Y C, B c l-x L$ (BCL2L1), MCL1, BIM (BCL2L11), HEXIM1/2, PIM1, BAD, $C D K N 1 A, I T G A M(C D 11 b)$, and $C E B P \alpha / \beta$, while reducing chromatin accessibility at cis-regulatory regions of other genes, including IL7 receptor (IL7R), MYB, and CDK6 (Fig. 1b). In BETi-treated SET2 cells, ATAC-Seqdetermined accessible chromatin demonstrated significant enrichment for the canonical binding sites of CTCF, TAL1/SCL, GATA2, RUNX1, ERG, c-Myc, and PU.1 (Fig. 1c). We also determined the status of H3K4Me3 and H3K27Ac marks at the promoter of MCL1 and $B c l-x L$ in OCI-AML5 or MV4-11 cells, utilizing ChIP-QPCR analyses. Treatment with ABBV-075 did not significantly alter the levels of these chromatin marks at the promoter of $M C L 1$ or $B c l-x L$ (Fig. S1).

Utilizing RNA-Seq analysis, we also determined the impact of BETi-induced perturbations in accessible chromatin on mRNA expressions in the AML SET2 cells. Figure $1 \mathrm{~d}$ shows the up- or down-regulated mRNA expressions in BETi-treated vs. untreated AML cells. Whereas HEXIM1 and CDKN1A (p21) mRNA levels were induced, mRNA levels of $M Y C, C D K 6, B C L 2 L 1$ (Bcl-xL), $B C L 2$, and PIM1 were downregulated. We next confirmed by qPCR analysis whether the more potent BETi ABBV075 induces similar mRNA perturbations in AML cells, including patient-derived (PD) CD34+ AML BPCs. As shown in Fig. 2a-d, ABBV-075 treatment attenuated $M Y C, B C L 2, B c l-x L$, and CDK6, while inducing HEXIM1 and $p 21$ mRNA levels in MV4-11, OCI-AML5, MOLM13, and PD CD34+ AML BPCs. Genetic alterations detected by NextGen sequencing (NGS) of an AML-associated 28gene panel, conducted in the AML cell lines, is presented in Fig. S2A. Utilizing a reversed phase protein array (RPPA) and Western analyses, we next determined the effect of ABBV-075 on protein expressions in AML BPCs. Figure S2B demonstrates the heat map of perturbations in protein levels by the RPPA analysis, showing increase in 29 and reduction in 55 protein expressions, following treatment of MV4-11 cells with ABBV-075 for $16 \mathrm{~h}$. As shown in Fig. S2C, among the log2-fold-altered protein levels, proteins involved in cell signaling, cell cycle, and transcription regulators were inhibited, while protein expressions involved in DNA damage response, cell cycle arrest, and cell death were increased. Western analyses confirmed that treatment with ABBV-075 attenuated protein expressions of c-Myc, CDK6, BCL2, and MCL1, while simultaneously inducing the levels of BRD4, HEXIM1, p21, p27, BIM and cleaved PARP in MV4-11, OCI-AML5, and MOLM13 cells (Fig. 2e, f and S2D). Notably, ABBV-075 treatment significantly reduced the expression of MCL1 in MV4-11 and OCI-AML5 AML cells (Fig. S3A and S3B) $(p<0.05)$. Following ABBV-075 treatment, similar effects on the protein levels were observed in three samples of PD CD34+ AML BPCs, including a decline in the levels of MCL1 (Fig. S3C to S3F). We also determined total and activated protein levels of BAX and BAK in AML cells. Whereas the total protein levels of BAX and BAK were unaffected (by Western analyses) (Fig. S4A), activated BAK levels in MOLM13 and activated BAX levels in OCI-AML5 cells were increased (Fig. S4B), following treatment with ABBV-075, as detected by flow cytometry after staining with antibodies that specifically detect the active conformation of BAK or BAX.

\section{ABBV-075 induces lethal effects in cultured AML cells lines and patient-derived AML blast progenitor cells}

We next determined the lethal effects of ABBV-075 in cultured AML cell lines and PD CD34+ AML BPCs. As shown in Fig. S4C and S4D, treatment with ABBV-075 dose-dependently induced apoptosis to a diverse extent in cultured AML cell lines, including FLT3-ITD and MLL fusion-protein expressing MV4-11 and MOLM13 cells. ABBV-075 treatment also induced apoptosis in OCI- 


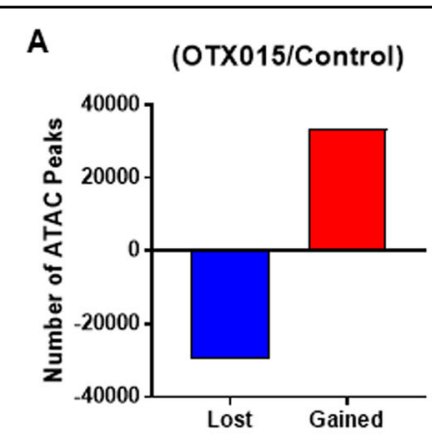

8

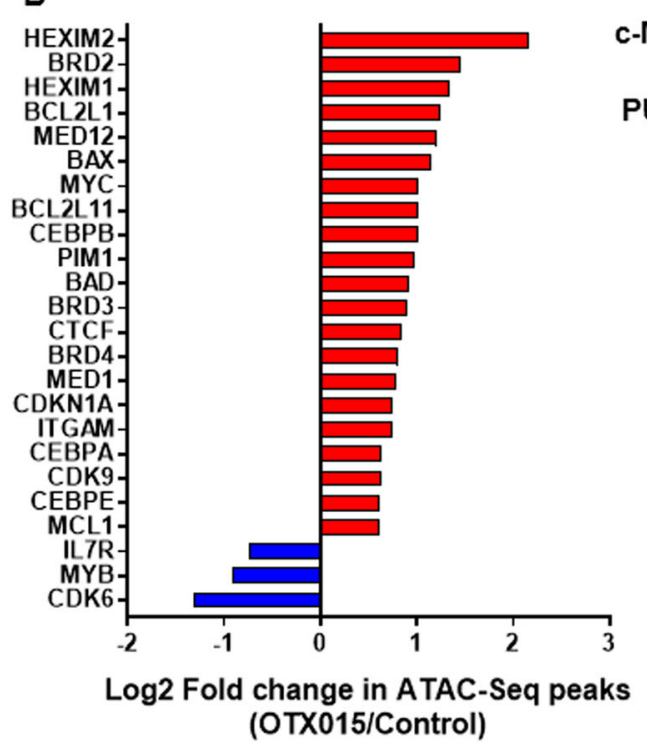

C TF Motif in ATAC peaks

CTCF ATAGTCCCACCT

TAL1/SCL

GATA2

RUNX1

ERG

c-Myc

PU.1

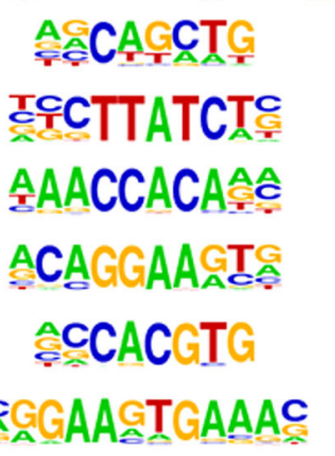

79.967

43.63

23.417

14.153

11.351

7.028

2.978

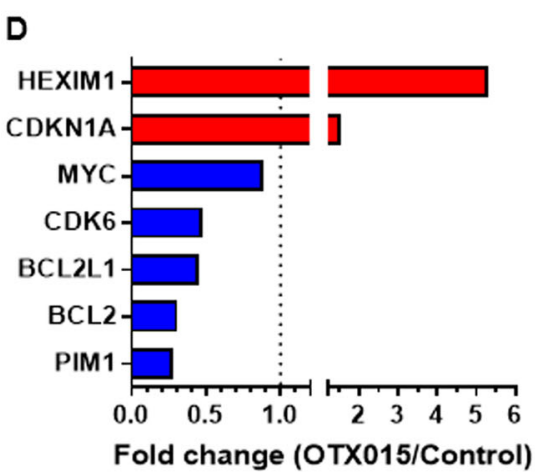

Fig. 1 Treatment with BET inhibitor alters chromatin accessibility and mRNA expression levels in AML cells. a AML SET2 cells were treated with BET inhibitor OTX015 for $16 \mathrm{~h}$. Cells were harvested and ATAC-Seq analysis was performed. The total number of peaks gained or lost in OTX015treated cells compared to untreated cells are shown. $\mathbf{b}$ Log2 fold-change in gained or lost peaks at the transcription start site or within $10 \mathrm{~kb}$ of the gene body of leukemia-relevant genes. c Transcription factor motifs increased in up (gained) peaks in OTX015-treated AML SET2 cells over control cells and the associated $-\log 10 p$ value. $\mathbf{d}$ Relative fold-change in BET inhibitor-treated AML SET2 cells over control as determined by RNA-Seq analysis

AML5, SKM1 and to a lesser extent in Mono-Mac-1 cells (Fig. S4D). Treatment with ABBV-075 also dosedependently induced lethality in 15 samples of PD CD34+ AML BPCs (seven untreated and eight treatmentrefractory) (Figs. S4E and S4F). Specific genetic alterations detected through NGS in these 15 AML samples is shown in Fig. S4G. No notable association was detected between any specific genetic alteration and ABBV-075-mediated loss-of-viability in the PD AML BPCs (Fig. S4E and S4F).

\section{Venetoclax (ABT-199) and A-1210477 increase MCL1 expression but induce apoptosis in AML cells}

We first determined the apoptotic effects of venetoclax alone in the cultured AML cell lines. As shown in Fig. 3a, venetoclax dose-dependently induced apoptosis in OCIAML5, MV4-11, and MOLM13 but not in SKM1 cells. Although venetoclax did not alter BCL2, BAX, and BAK levels, it did increase activated BAX and BAK levels in MOLM13 and OCI-AML5 cells (Fig. 3b, c and S5A). Notably, venetoclax treatment significantly increased MCL1 levels in these AML cell types (Fig. 3b, S5B and $\mathrm{S} 5 \mathrm{C})$. However, venetoclax simultaneously decreased Bcl$\mathrm{xL}$ levels, while increasing NOXA levels, which has been shown to counter the antiapoptotic activity of MCL1 ${ }^{22-24}$ (Fig. 3b and S5B). We next determined the activity of the MCL1 inhibitor A-1210477 against AML BPCs. As shown in Fig. 3d, exposure to A-1210477 dose-dependently induced apoptosis of MOLM13 and MV4-11, but not of OCI-AML5 and SKM1 cells. As previously reported, treatment with A-1210477 increased MCL1 expression by

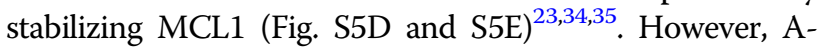
1210477 treatment also reduced $\mathrm{Bcl}-\mathrm{xL}$ and increased active BAK levels in MOLM13 and MV4-11 cells, without a similar effect in OCI-AML5 and SKM1 cells (Fig. S5D-S5F, 


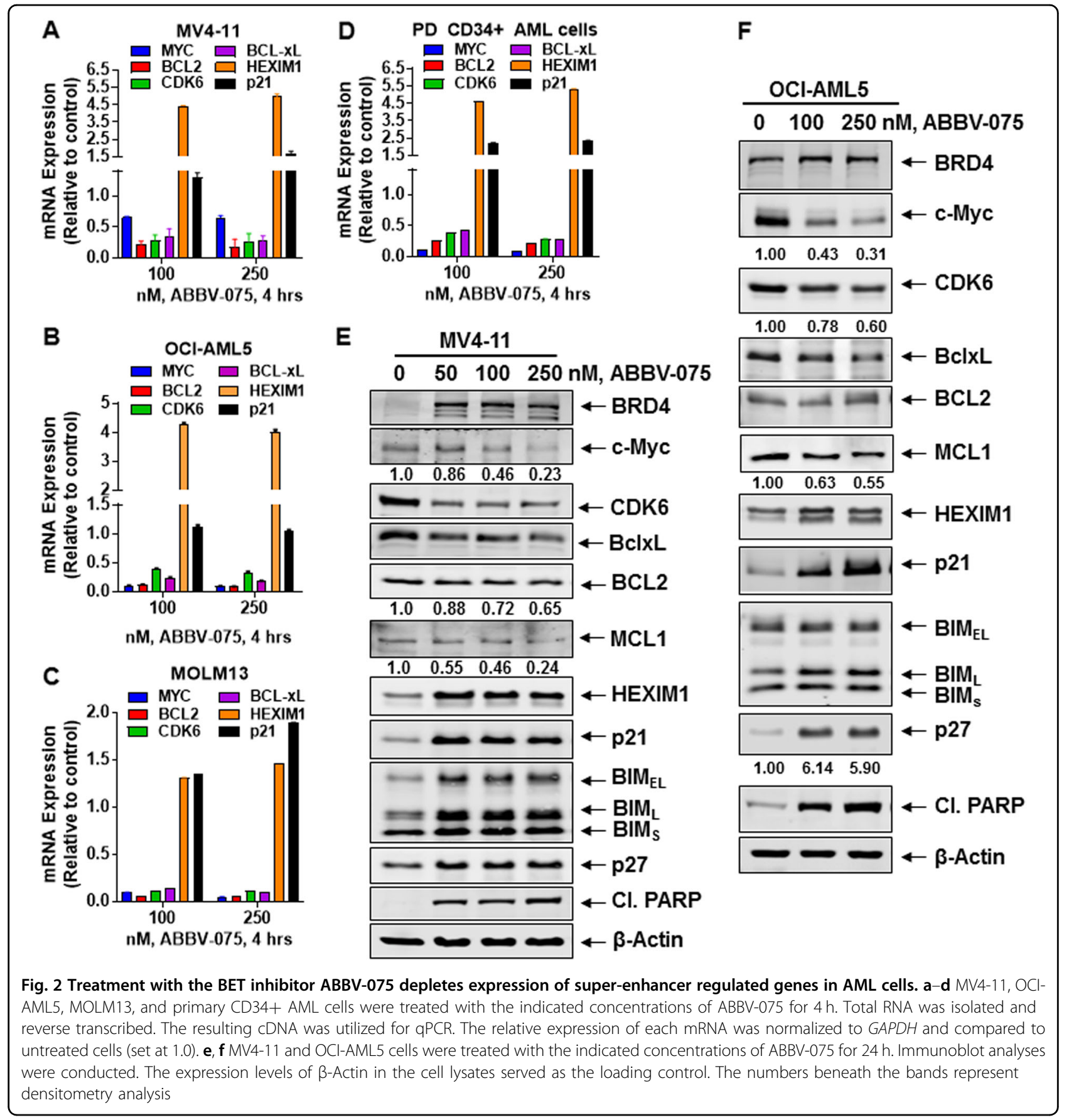

$\mathrm{S} 5 \mathrm{H}$, and data not shown), which may partially explain A1210477-induced apoptosis in MOLM13 and MV4-11 but not in OCI-AML5 and SKM1 cells (Fig. 3d).

Cotreatment with ABBV-075 and venetoclax or A-1210477 synergistically induces in vitro lethality in AML cells

We first determined the in vitro lethal activity of combined exposure to various concentrations of ABBV-075 and venetoclax for $48 \mathrm{~h}$, as compared to each agent alone, in AML cells (Fig. S6A to S6D). Notably, cotreatment with
ABBV-075 and venetoclax synergistically induced apoptosis of MOLM13, MV4-11, and OCI-AML5 cells (CI values of <1.0) (Fig. 4a). The combination was also synergistically lethal against SKM1 cells, which were resistant to apoptosis induced by venetoclax alone (Figs. $3 \mathrm{a}$ and $4 \mathrm{a})$. ABBV-075 treatment reduced the levels of MCL1, Bcl-xL, MYC, and CDK6, while increasing HEXIM1, p21, p27, and cleaved PARP levels in SKM1 cells (Fig. S5G). The combination of ABBV-075 and venetoclax was also effective in reducing MCL1, Bcl- 

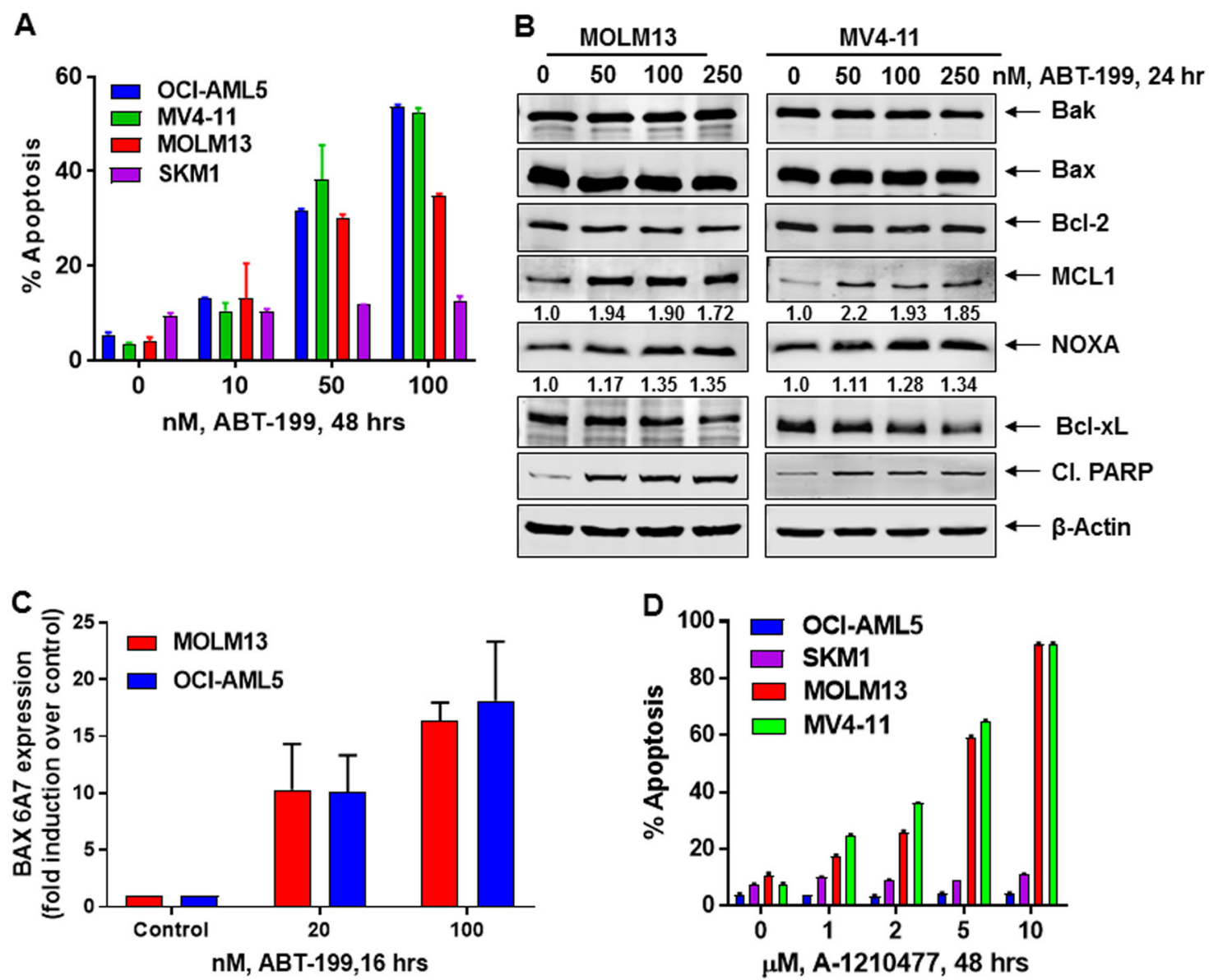

Fig. 3 Treatment with ABT-199 (venetoclax) or MCL1 inhibitor A-1210477 induces apoptosis of AML cells. a OCI-AML5, MV4-11, MOLM13, and SKM1 cells were treated with the indicated concentrations of ABT-199 for $48 \mathrm{~h}$. Then, the $\%$ of annexin V-positive, apoptotic cells were determined by flow cytometry. Columns, mean of three experiments; Bars, Standard error of the mean. b MOLM13 and MV4-11 cells were treated with the indicated concentrations of ABT-199 for $24 \mathrm{~h}$. Total cell lysates were prepared and immunoblot analysis was conducted. The expression levels of $\beta$-Actin in the cell lysates served as the loading control. The numbers beneath the bands represent densitometry analysis. c MOLM13 and OCI-AML5 cells were treated with the indicated concentrations of ABT-199 for $16 \mathrm{~h}$. Cells were fixed, permeabilized and stained with Alexa488conjugated BAX (6A7) antibody followed by flow cytometry. Values represent fold induction of active BAX over control cells. d OCI-AML5, MV4-11, MOLM13, and SKM1 cells were treated with the indicated concentrations of the MCL1-specific inhibitor, A-1210477 for $48 \mathrm{~h}$. The \% of annexin V-positive, apoptotic cells were determined by flow cytometry. Columns, mean of three experiments; Bars, Standard error of the mean

$\mathrm{xL}$, and BCL-2 protein levels in MV4-11 and OCI-AML5 cells (Fig. 4b, c). Although protein levels of BAX and BAK were unaltered (Fig. S7A), cleaved PARP levels increased significantly in association with the synergistic induction of apoptosis (Fig. 4b, c). Cotreatment with ABBV-075 and venetoclax also markedly reduced MYC and CDK6 levels, undermining their likely progrowth and prosurvival effects in the AML cells (Fig. 4b, c). We next determined the effects of combined treatment with ABBV-075 and A1210477 in the AML cells. As shown, coexposure to ABBV-075 and A-1210477 at various concentrations synergistically induced apoptosis not only in MOLM13 and MV4-11 but also in OCI-AML5 and SKM1 cells (CI values of <1.0) (Fig. 4d and S7B to S7E), which were resistant to apoptosis induced by A-1210477 alone (Fig. 3d). This may not only be because ABBV-075 treatment alone reduces MCL1, BCL2, MYC, and CDK6 levels, but also because it increases p21, p27, and HEXIM1 levels in AML cells (Fig. 2e, f and S2D). Additionally, cotreatment with A-1210477 and ABBV-075, vs. treatment with each drug alone, caused greater induction in $\mathrm{p} 21$, $\mathrm{BIM}$, and cleaved PARP levels, while attenuating c-Myc levels in OCI-AML5 cells (Fig. S7F). We also determined the lethal activity of cotreatment with venetoclax and A1210477 at various concentrations in AML cells (Fig. S8A). As shown, cotreatment with venetoclax and A-1210477 synergistically induced apoptosis of MOLM13, MV4-11, SKM1, and OCI-AML5 cells (CI values of $<1.0)$ (Fig. S8B). 

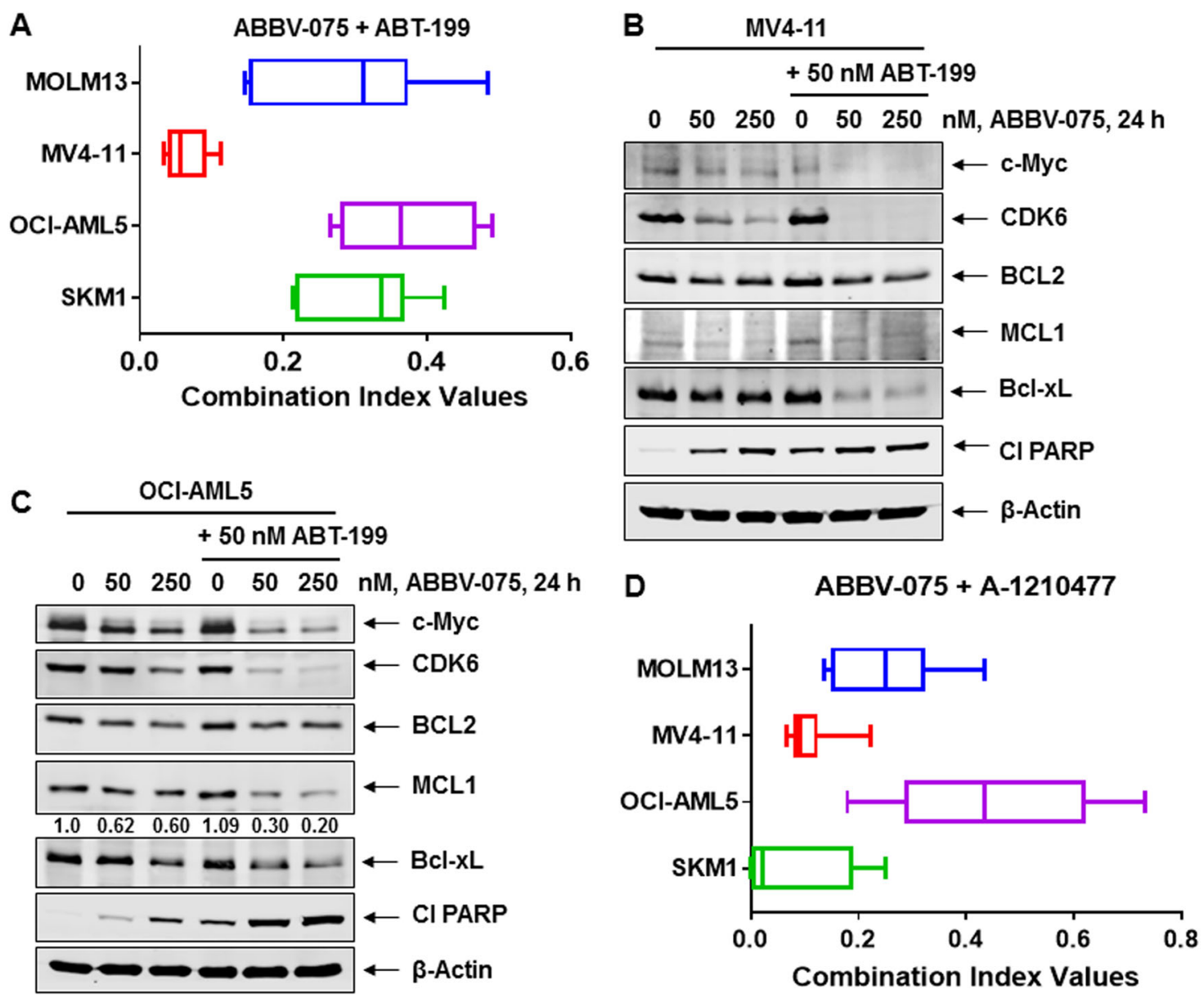

Fig. 4 Cotreatment with ABBV-075 and ABT-199 or MCL1 inhibitor A-1210477 exerts synergistic lethal activity against AML cells. a MOLM13, MV4-11, OCI-AML5, and SKM1 cells were treated with ABBV-075 (dose range: 20-250 nM) and ABT-199 (dose range: 10-100 nM) for 48 h. Following this, the \% of annexin V-positive, apoptotic cells was determined by flow cytometry. Combination index values were calculated by Compusyn and graphed utilizing GraphPad V7. b, c MV4-11 and OCI-AML5 cells were treated with the indicated concentrations of ABBV-075 and/or ABT-199 for $24 \mathrm{~h}$. Total cell lysates were prepared and immunoblot analyses were conducted. The expression levels of $\beta$-Actin in the cell lysates served as the loading control. d MOLM13, MV4-11, OCI-AML5, and SKM1 cells were treated with ABBV-075 (dose range: 20-250 nM) and A-1210477 (dose range: $1-10 \mu \mathrm{M}$ ) for $48 \mathrm{~h}$. Following this, the \% of annexin V-positive, apoptotic cells was determined by flow cytometry. Combination index values were determined by Compusyn and graphed utilizing GraphPad V7

Cotreatment with ABBV-075 and venetoclax or A-1210477 synergistically induces in vitro lethality in PD CD34+ AML BPCs

Utilizing the 15 samples of PD CD34+ AML BPCs, exhibiting genetic mutations shown in Fig. S4G, we first determined the lethal effects of exposure to venetoclax alone over $48 \mathrm{~h}$. In a dose-dependent manner, venetoclax treatment significantly increased loss-of-viability in these cells (Fig. 5a). Notably, exposure to a range of concentrations of ABBV-075 and venetoclax synergistically induced apoptosis in the 15 samples of CD34+ AML BPCs (CI values < 1.0) (Fig. $5 \mathrm{~b}$ and S9). We also determined the lethal activity of cotreatment with a range of concentrations of ABBV-075 and A-1210477 in five of the samples (\#14-18) of PD CD34+ AML BPCs (Figs. S4G and S10). As shown in Fig. 5c, cotreatment with ABBV075 and A-1210477 synergistically induced loss-ofviability in the $\mathrm{AML} \mathrm{BPCs}(\mathrm{CI}$ values $<1.0)$. It is also noteworthy that the combination of ABT-199 and A1210477, involving exposure to concentrations of each drug documented in Fig. S11 also synergistically induced loss-of-viability in three samples of PD CD34+ AML BPCs (Fig. 5d and S4G). We also determined the lethal activity of cotreatment with ABBV-075 and ABT-199 or A-1210477 against normal CD34+ HPCs. As shown in Fig. S12A and S12B, cotreatment with ABBV-075 and ABT-199 or A-1210477 did not induce significant loss-ofviability in normal CD34+ HPCs. 


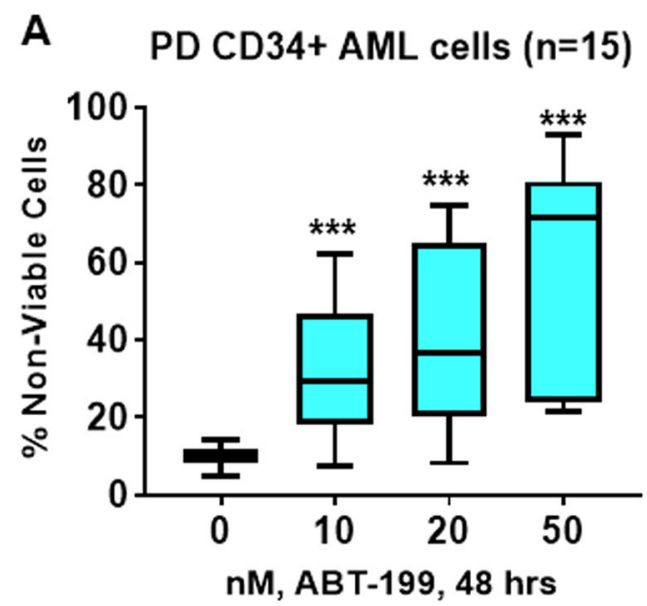

C

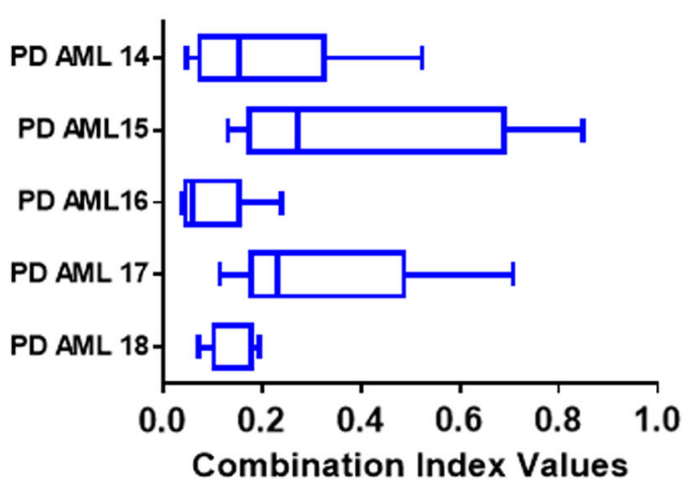

B

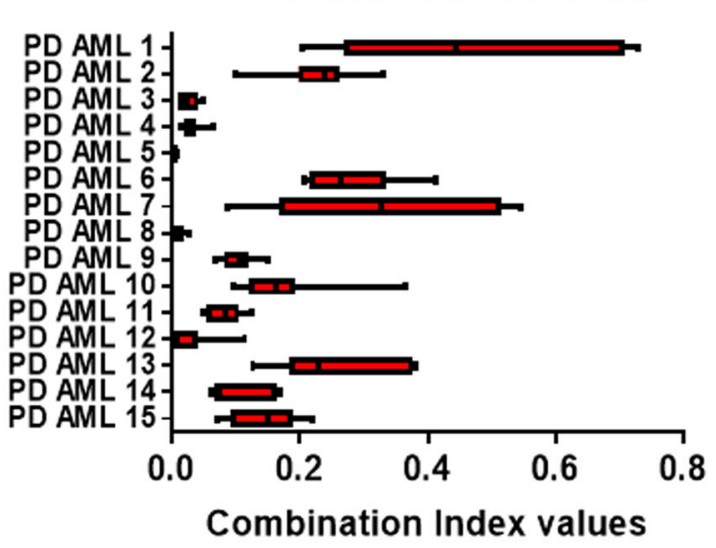

A-1210477 and ABT-199

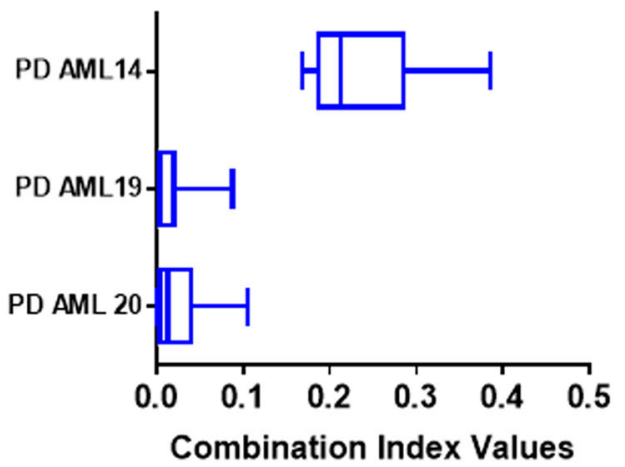

Fig. 5 Cotreatment with ABBV-075 and ABT-199 or MCL1 inhibitor exerts synergistic lethal activity against patient-derived (PD) AML BPCs. a PD, CD34+ AML BPCs were treated with the indicated concentrations of ABT-199 for $48 \mathrm{~h}$. At the end of treatment, cells were stained with To-Pro-3 iodide and the $\%$ of nonviable cells were determined by flow cytometry. A box plot was generated utilizing GraphPad V7. ${ }^{* * *}=p<0.005$ compared to the untreated control cells. $\mathbf{b}$ PD, CD34+ AML BPCs $(n=15)$ were treated with ABBV-075 (dose range: 20-250 nM) and ABT-199 (dose range: 10-100 nM) for $48 \mathrm{~h}$. Following this, the \% of nonviable cells was determined by flow cytometry. Combination index values were calculated by Compusyn and graphed utilizing GraphPad V7. c PD, CD34+ AML BPCs $(n=5)$ were treated with ABBV-075 (dose range: 20-250 nM) and A-1210477 (dose range: 1-10 $\mu \mathrm{M}$ ) for $48 \mathrm{~h}$. Following this, the $\%$ of nonviable cells was determined by flow cytometry. Combination index values were calculated by Compusyn and graphed utilizing GraphPad V7. d PD CD34+ AML BPCs $(n=3)$ were treated with A-1210477 (dose range: 2-10 $\mu \mathrm{M})$ and ABT-199 (dose range: 10-50 nM) for $48 \mathrm{~h}$. Following this, the \% of nonviable cells was determined by flow cytometry. Combination index values were calculated by Compusyn and graphed utilizing GraphPad V7. BPC blast progenitor cell

Superior in vivo activity of cotreatment with ABBV-075 and venetoclax vs. each agent alone against $A M L$ cells

We next determined the in vivo anti-AML activity of ABBV-075 and/or venetoclax against the MOLM13/GFPLuc cell xenograft in NSG mice. Five days after tail vein infusion and engraftment of MOLM13/GFP-Luc cells, treatment with vehicle alone or ABBV-075 and/or venetoclax was started. As shown in Fig. 6a, as compared to treatment with vehicle control or ABBV-075 alone, treatment with venetoclax alone or cotreatment with ABBV-075 and venetoclax was more effective in reducing the AML xenograft growth 1-week post-engraftment of MOLM13/GFP-Luc cells (Fig. 6a, b). Combined treatment with ABBV-075 and venetoclax, vs. each agent alone also significantly improved the median and overall survival of
NSG mice engrafted with MOLM13/GFP-Luc cells $(p<$ 0.001 ), as shown in the Kaplan-Meier plot depicting the survival of the mice (Fig. 6b). Notably, as compared to vehicle control or ABBV-075 alone, cotreatment with ABBV-075 and venetoclax was also significantly more effective in reducing growth of a PDX model of AML cells $(45, \mathrm{XX}, \mathrm{t}(3 ; 12)$ (q26.1;p13),-7 with NRAS mutation) engrafted in NSG mice (Fig. 6c).

\section{Discussion}

It is well-documented that $\mathrm{BH} 3$ domain-only proteins, e.g., BIM and NOXA, either directly induce and activate $\mathrm{BAX} / \mathrm{BAK}$ oligomers or release them from antiapoptotic BCL2, MCL1, and Bcl-xL to trigger mitochondrial outer membrane permeabilization (MOMP) and the ensuing 

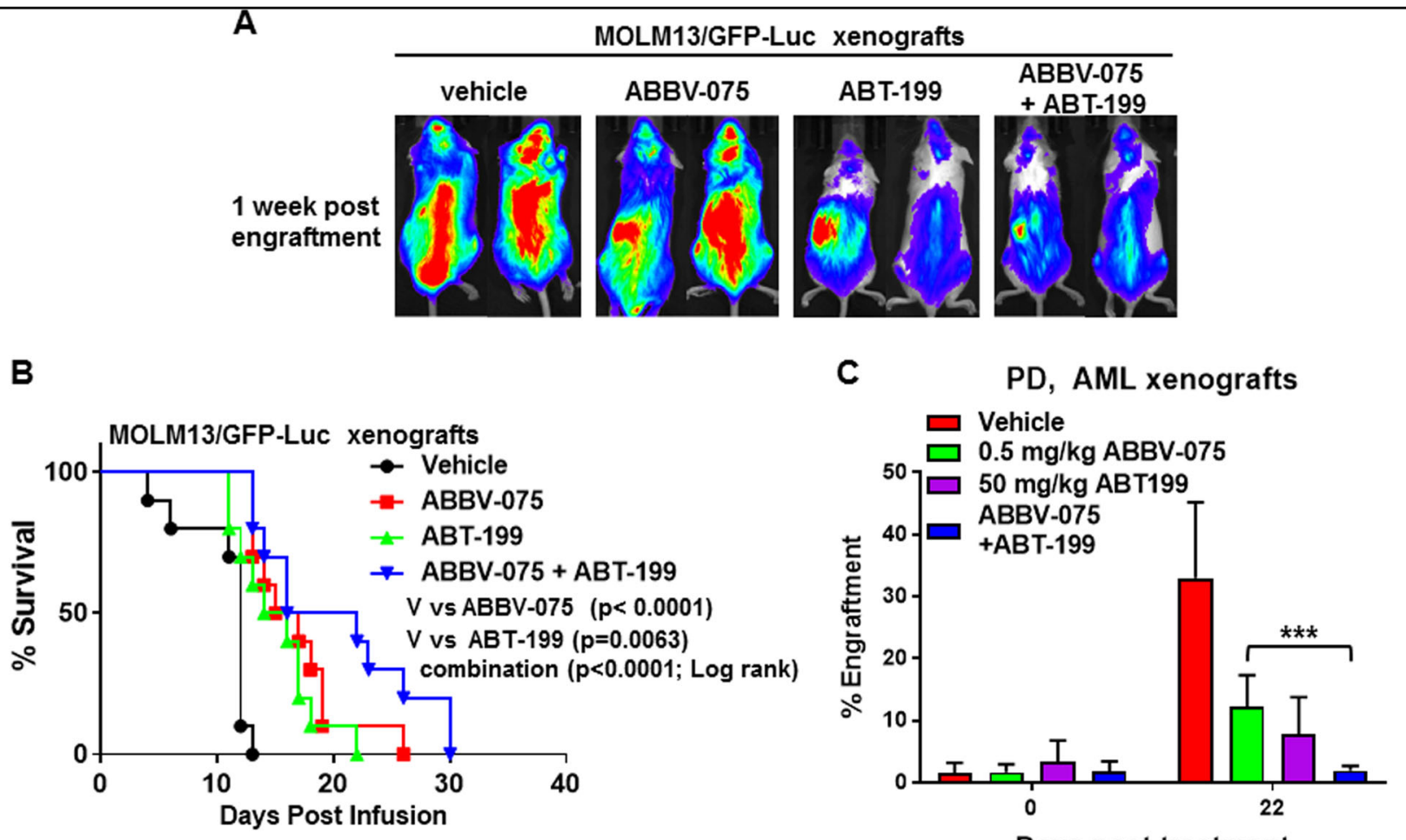

Days post treatment

Fig. 6 Cotreatment with ABBV-075 and ABT-199 improves survival of NSG mice-bearing AML xenografts. a MOLM13/GFP-LUC cells $\left(2 \times 10^{6}\right)$ were introduced into the tail vein of pre-irradiated (2.5 Gy) NSG mice ( $n=7$ per cohort). Mice were monitored for 4 days and then treated with ABBV075 and/or ABT-199 for 2 weeks. Mice were imaged after 1 week of treatment utilizing a xenogen camera. b Kaplan-Meier survival curve of NSG mice-bearing MOLM13/GFP-Luc xenografts treated with ABBV-075 and/or ABT-199. c Patient-derived AML xenografts (PDX) were implanted into NSG mice ( $n=8$ mice per cohort). Following documentation of engraftment, mice were treated with the indicated doses of ABBV-075 and/or ABT-199 for 22 days. Following this, the $\%$ of AML engraftment in the mice was determined by flow cytometry. ${ }^{* *}=p<0.005$ compared to ABBV-075-treated mice. NSG NOD-scid IL2Rgamma ${ }^{\text {null }}$

execution phase of apoptosis in AML BPCs ${ }^{22-24}$. Therefore, prosurvival BCL2, MCL1, and Bcl-xL bind and sequester BAX/BAK and BIM/NOXA to inhibit the commitment and execution of apoptosis in a BAX/BAKdependent manner in AML $\mathrm{BPC}^{22-25,36}$. Mitochondrial priming represents how close a leukemia cell is to apoptosis threshold and how addicted it is to specific antiapoptotic BCL-2 protein family members for survival ${ }^{36-38}$. Although not prospectively validated in the clinical setting, BH3-profiling has revealed mitochondrial priming and cellular addiction to prosurvival BCL2 or MCL1 in AML cells ${ }^{26,36,38,39}$. In contrast, antiapoptotic potency of BCL2 family of proteins was shown to primarily depend upon their stability and not on binding selectivity with the proapoptotic members ${ }^{40}$. Recently, measurements of cellular tolerance to levels of $\mathrm{BH} 3$ domain-only proteins, based on relative contributions of anti- and proapoptotic protein family members, was shown to predict susceptibility to apoptosis induced by antileukemia drugs and combinations $^{41}$. Consistent with above, previous reports have highlighted that increase in levels and addiction to MCL1 and/or Bcl-xL confers innate or acquired resistance to venetoclax in leukemia and lymphoma cells (Fig. 7) ${ }^{29,30}$. This could be overcome by simultaneously targeting MCL1 and/or Bcl-xL (Fig. 7) ${ }^{29-31,42,43}$. Here, in AML BPCs, we demonstrate for the first time that treatment with the BETi ABBV-075 reduces MCL1 and Bcl-xL levels, while inducing BIM levels, thereby increasing activated BAX and/or BAK levels and undermining the role of MCL1 and Bcl-xL in mediating resistance to venetoclax-induced apoptosis (Fig. 7). This is further supported by our findings that whereas SKM1 cells were resistant to apoptosis induced by venetoclax alone, they were sensitive to ABBV-075 alone, and cotreatment with ABBV-075 and venetoclax synergistically induced apoptosis of SKM1 cells. Additionally, since similar to other BETis, ABBV-075 treatment attenuated MYC and CDK6, while simultaneously inducing HEXIM1 and CDKN1A $(\mathrm{p} 21)^{11,12,44-47}$; these perturbations also likely augmented venetoclax-mediated inhibition of growth and survival of AML BPCs. Notably, the synergistic lethal activity of cotreatment with ABBV-075 and venetoclax was observed not only against cultured cell lines but also against PD $\mathrm{CD} 34+\mathrm{BPCs}$ exhibiting diverse genetic alterations. It is 


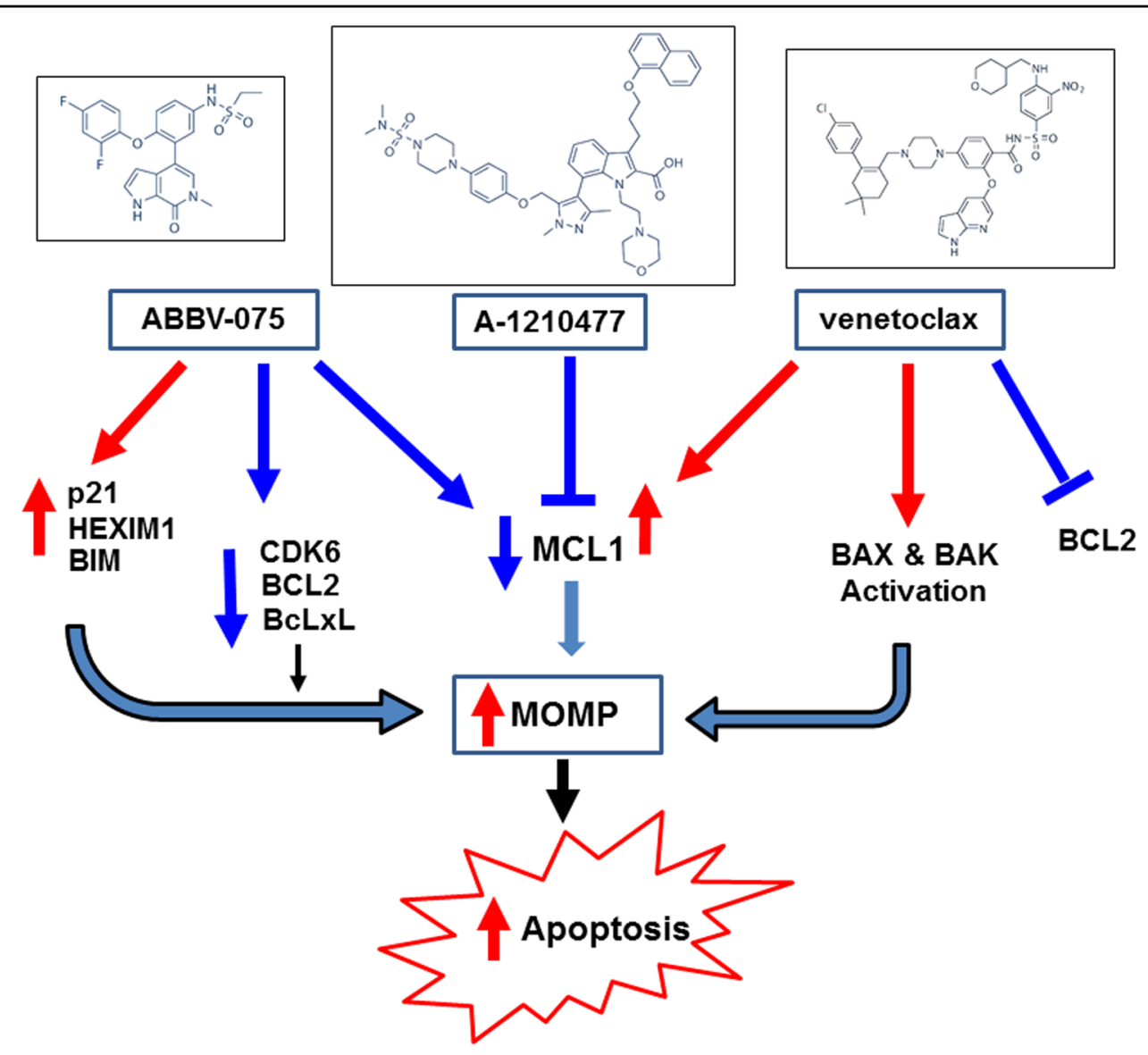

Fig. 7 Proposed model of the antileukemia activity of ABBV-075 and ABT-199 or A-1210477 against AML cells. ABBV- 075 depletes the expression of MCL1 and super-enhancer-driven oncogenes CDK6, BCL2 and BCIXL with concomitant induction of the expression of p21, HEXIM1, and BIM in the AML cells. This leads to increased mitochondrial outer membrane permeabilization (MOMP) and induction of apoptosis in the AML cells. Treatment with A-1210477 blocks the activity of MCL1 in the AML cells which also leads to increased MOMP and induction of apoptosis. Treatment with venetoclax inhibits the activity of BCL2 and activates conformation change of BAX and BAK leading to increased MOMP and induction of apoptosis. Cotreatment with ABBV-075 and ABT-199 or A-1210477 or ABT-199 and A-1210477 exerts synergistic lethal activity resulting in greater apoptosis in AML cells than treatment with the single agents

also noteworthy that the concentrations of ABBV-075 and venetoclax utilized in present studies demonstrating synergy of the combination are achievable in vivo and clinically and therefore relevant ${ }^{46,48}$. Additionally, the dose of each drug used alone and in combination in the vivo studies was safe and exhibited superior efficacy. Recently, a mutation in the BH3-peptide-binding hydrophobic pocket of BCL2 that reduces venetoclax binding has been shown to confer resistance to venetoclax in cultured cell lines ${ }^{29,49}$. However, the clinical relevance of this mechanism of resistance is uncertain, since it has not been documented in PD AML BPCs. Taken together, our findings support further clinical evaluation of combination therapy of AML with ABBV-075 and venetoclax. Previous clinical studies of BCL2/Bcl-xL inhibitor navitoclax in patients with CLL and small cell lung cancer demonstrated thrombocytopenia as a major dose-limiting toxicity, which would militate against the use of navitoclax in combination with ABBV-075 $5^{50,51}$.

Consistent with previous reports, our findings also show that A-1210477 treatment increased MCL1 levels ${ }^{34,35}$. Stability of MCL1 is controlled by ubiquitylation by the E3 ligase MULE (MCL1 ubiquitin ligase E3) and deubiquitinase USP9 $\mathrm{X}^{52,53}$. By displacing $\mathrm{BH} 3$ domain-containing MULE from MCL1, A-1210477 is known to stabilize and increase MCL1 levels ${ }^{23,34,35}$. However, as shown here and previously in other reports, treatment with $\mathrm{BETi}$, including ABBV-075, inhibits RNAP2-mediated transcription and levels of MCL1 and Bcl-xL, while simultaneously inducing BIM levels ${ }^{12,17,44,46}$. Together these perturbations due to superior lethal activity of cotargeting MCL1 and BCL2 is also underscored here by our findings that cotreatment with A-1210477 and venetoclax induces synergistic lethal effects against AML BPCs. Similar to the 
effects of ABBV-075, previous reports have also documented that cotreatment with CDK9 inhibitor that transcriptionally reduces short-lived MCL1 levels also enhances lethal effects of BCL2 inhibition ${ }^{54}$.

Several recent reports have also presented evidence for superior activity of combining venetoclax with other conventional or novel anti-AML agents, besides the BETi described here, that further reduce the threshold for apoptosis in AML BPCs. These include combinations with standard anti-AML agents, e.g., azacytidine ${ }^{32}$, cytarbine and idarubicin ${ }^{30}$, which induce mitochondrial priming by inducing the expressions of the $\mathrm{BH} 3$ domainonly proapoptosis proteins such as BIM, NOXA, and PUMA in a p53-dependent or independent manner ${ }^{41,55,56}$. Combinations of venetoclax with novel anti-AML agents include inhibitors targeting MEK, PI3K/mTOR, NEDD8, HDM2, and IDH1/25 $2^{5759}$. There is evidence to suggest that venetoclax may selectively target AML stem cells that depend on mitochondrial oxidative phosphorylation ${ }^{60}$. If so, how combining each of these novel agents modulates this important activity of venetoclax would have to be evaluated in future studies.

\section{Data availability}

RNA-Seq datasets are deposited in GEO under accession number (GSE93578). Methods for sequencing of primary AML samples, reverse phase protein array, ATAC-Seq analysis and assessment of BAX and BAK conformation change are provided in the Supplemental Methods.

\section{Acknowledgements}

The authors would like to thank the Sequencing and Microarray Core Facility, Flow Cytometry and Cellular Imaging (FCCl) Core Facility and the Functional Proteomics RPPA Core facility which are supported by the MD Anderson Cancer Center Support Grant 5P30 CA016672-40. The heat maps were developed by the MD Anderson Cancer Center Department of Bioinformatics and Computational Biology, In Silico Solutions, Santeon and SRA International. This work was supported in part by U.S. National Cancer Institute (NCl; MD Anderson TCGA Genome Data Analysis Center) grant numbers CA143883 and CA083639, the Mary K. Chapman Foundation, the Michael \& Susan Dell Foundation (honoring Lorraine Dell), and MD Anderson Cancer Center Support Grant P30 CA016672 (the Bioinformatics Shared Resource). This project was partially supported by CPRIT RP170295 (C.C.), the shared Proteomics and Metabolomics core at Baylor College of Medicine with funding from the $\mathrm{NIH}$ (P30 CA125123), CPRIT Proteomics and Metabolomics Core Facility RP170005 (K.R., C.C.), and the NCl-recognized Dan L. Duncan Cancer Center. This research is supported in part by the MD Anderson Cancer Center Leukemia SPORE (P50 CA100632).

\section{Author details}

${ }^{1}$ The University of Texas MD Anderson Cancer Center, Houston, TX 77030, USA. ${ }^{2}$ Department of Molecular and Cellular Biology, Baylor College of Medicine, Houston, TX 77030, USA. ${ }^{3}$ AbbVie, Inc., North Chicago, IL 60064, USA

\section{Authors' contributions}

Conceptualization, K.N.B.; Formal analysis, C.C., K.R. and D.P.; Investigation, W.F., T.C., C.P.M., D.T.S., B.S., D.N.S., M.K.; Resources, C.D.D., S.M.K., G.B., T.M.K., N.P., P.B., L.M., J.D.K., Y.S.; Visualization, W.F., C.P.M., C.C. and K.R.; Writing-Original draft, K.N.B., W.F.; Writing-Review and editing, K.N.B.

\section{Conflict of interest}

Y.S. is an employee of AbbVie, Inc. All other authors declare that they have no conflict of interest.

\section{Publisher's note}

Springer Nature remains neutral with regard to jurisdictional claims in published maps and institutional affiliations.

Supplementary Information accompanies this paper at (https://doi.org/ 10.1038/s41408-018-0165-5).

Received: 5 September 2018 Revised: 25 November 2018 Accepted: 28 November 2018

Published online: 15 January 2019

\section{References}

1. Belkina, A. C. \& Denis, G. V. BET domain co-regulators in obesity, inflammation and cancer. Nat. Rev. Cancer 12, 465-477 (2012).

2. Shi, J. \& Vakoc, C. R. The mechanism behind the therapeutic activity of BET bromodomain inhibition. Mol. Cell 54, 728-736 (2014).

3. Itzen, F., Greifenberg, A. K., Bosken, C. A. \& Geyer, M. Brd4 activates P-TEFB for RNA polymerase II CTD phosphorylation. Nucleic Acids Res. 42, 7577-7590 (2014).

4. Nechaev, S. \& Adelman, K. Pol II waiting in the starting gates: regulating the transition from transcription initiation into productive elongation. Biochim. Biophys. Acta 1809, 34-45 (2011).

5. Winter, G. E. et al. BET bromodomain proteins function as master transcription elongation factors independent of CDK9 recruitment. Mol. Cell 67, 5-18.e19 (2017).

6. Bradner, J. E., Hnisz, D. \& Young, R. A. Transcriptional addiction in cancer. Cell 168, 629-643 (2017).

7. Loven, J. et al. Selective inhibition of tumor oncogenes by disruption of superenhancers. Cell 153, 320-334 (2013).

8. Hnisz, D. et al. Convergence of developmental and oncogenic signaling pathways at transcriptional superenhancers. Mol. Cell 58, 362-370 (2015).

9. Bhagwat, A. S., Lu, B. \& Vakoc, C. R. Enhancer dysfunction in leukemia. Blood 131, 1795-1804 (2018).

10. Zuber, J. et al. RNAi screen identifies Brd4 as a therapeutic target in acute myeloid leukaemia. Nature 478, 524-528 (2011).

11. Dawson, M. A. et al. Inhibition of BET recruitment to chromatin as an effective treatment for MLL-fusion leukaemia. Nature 478, 529-533 (2011).

12. Fiskus, W. et al. Highly active combination of BRD4 antagonist and histone deacetylase inhibitor against human acute myelogenous leukemia cells. Mol. Cancer Ther. 13, 1142-1154 (2014).

13. Dawson, M. A. et al. Recurrent mutations, including NPM1c, activate a BRD4dependent core transcriptional program in acute myeloid leukemia. Leukemia 28, 311-320 (2014)

14. Berthon, C. et al. Bromodomain inhibitor OTX015 in patients with acute leukaemia: a dose-escalation, phase 1 study. Lancet Haematol. 3, e186-e195 (2016).

15. Braun, T. \& Gardin, C. Investigational BET bromodomain protein inhibitors in early stage clinical trials for acute myelogenous leukemia (AML). Expert Opin. Investig. Drugs 26, 803-811 (2017).

16. Filippakapoulos, P. \& Knapp, S. Targeting bromodomains: epigenetic readers of lysine acetylation. Nat. Rev. Drug Discov. 13, 337-356 (2014).

17. Stathis, A. \& Bertoni, F. BET proteins as targets for anticancer treatment. Cancer Discov. 8, 24-36 (2018).

18. McDaniel, K. F. et al. Discovery of N-(4-(2,4-Difluorophenoxy)-3-(6-methyl-7oxo-6,7-dihydro-1H-pyrrolo[2,3-c] pyridin-4-yl)phenyl)ethanesulfonamide (ABBV-075/Mivebresib), a potent and orally available bromodomain and extraterminal domain (BET) family bromodomain inhibitor. J. Med. Chem. 60, 8369-8384 (2017)

19. Tanaka, M. et al. Design and characterization of bivalent BET inhibitors. Nat. Chem. Biol. 12, 1089-1096 (2016).

20. Rhyasen, G. W. et al. AZD5153: a novel bivalent BET bromodomain inhibitor highly active against hematologic malignancies. Mol. Cancer Ther. 15, 2563-2574 (2016).

21. Hogg, S. J. et al. BET inhibition induces apoptosis in aggressive B-cell lymphoma via epigenetic regulation of BCL-2 family members. Mol. Cancer Ther. 15, 2030-2041 (2016).

22. Adams, J. M. \& Cory, S. The Bcl-2 apoptotic switch in cancer development and therapy. Oncogene 26, 1324-1337 (2007). 
23. Ashkenazi, A., Fairbrother, W. J., Leverson, J. D. \& Souers, A. J. From basic apoptosis discoveries to advanced selective BCL-2 family inhibitors. Nat. Rev. Drug Discov. 16, 273-284 (2017).

24. Dai, H., Meng, X. W. \& Kaufmann, S. H. Mitochondrial apoptosis and BH3 mimetics. Flo00Res. 5, 2804-2815 (2016).

25. Leverson, J. D. et al. Found in translation: how preclinical research is guiding the clinical development of the BCL2-selective inhibitor venetoclax. Cancer Discov. 7, 1376-1393 (2017).

26. Pan, R. et al. Selective BCL-2 inhibition by ABT-199 causes on-target cell death in acute myeloid leukemia. Cancer Discov. 4, 362-375 (2014).

27. Souers, A. J. et al. ABT-199, a potent and selective BCL-2 inhibitor, achieves antitumor activity while sparing platelets. Nat. Med. 19, 202-208 (2013).

28. Konopleva, M. et al. Efficacy and biological correlates of response in a phase II study of venetoclax monotherapy in patients with acute myelogenous leukemia. Cancer Discov. 6, 1106-1117 (2016).

29. Tahir, S. K. et al. Potential mechanisms of resistance to venetoclax and strategies to circumvent it. Bmc Cancer 17, 399 (2017).

30. Teh, T. C. et al. Enhancing venetoclax activity in acute myeloid leukemia by cotargeting MCL1. Leukemia 32, 303-312 (2018).

31. Phillips, D. C. et al. Loss in MCL1 function sensitizes non-Hodgkin's lymphoma cell lines to the BCL-2-selective inhibitor venetoclax (ABT-199). Blood Cancer J. 5, e368 (2015).

32. Bogenberger, J. M. et al. BCL-2 family proteins as 5-Azacytidine-sensitizing targets and determinants of response in myeloid malignancies. Leukemia $\mathbf{2 8}$ 1657-1665 (2014).

33. DiNardo, C. D. et al. Clinical experience with the BCL2-inhibitor venetoclax in combination therapy for relapsed and refractory acute myeloid leukemia and related myeloid malignancies. Am. J. Hematol. 93, 401-407 (2018).

34. Leverson, J. D. et al. Potent and selective small-molecule MCL1 inhibitors demonstrate on-target cancer cell killing activity as single agents and in combination with ABT-263 (navitoclax). Cell Death Dis. 6, e1590 (2015).

35. Soderquist, S., Eastman, R. \& A. BCL2 inhibitors as anticancer drugs: a plethora of misleading BH3 mimetics. Mol. Cancer Ther. 15, 2011-2017 (2016).

36. Valentin, R., Grabow, S. \& Davids, M. S. The rise of apoptosis: targeting apoptosis in hematologic malignancies. Blood 132, 1248-1264 (2018).

37. Certo, M. et al. Mitochondria primed by death signals determine cellular addiction to antiapoptotic BCL-2 family members. Cancer Cell 9, 351-365 (2006).

38. Vo, T. T. et al. Relative mitochondrial priming of myeloblasts and normal HSCs determines chemotherapeutic success in AML. Cell 151, 344-355 (2012).

39. Ni Chonghaile, T. et al. Pretreatment mitochondrial priming correlates with clinical response to cytotoxic chemotherapy. Science 334, 1129-1133 (2011).

40. Rooswinkel, R. W. et al. Antiapoptotic potency of $\mathrm{BCl}-2$ proteins primarily relies on their stability, not binding selectivity. Blood 123, 2806-2815 (2014).

41. Dai, H. et al. Measurement of BH3-only protein tolerance. Cell Death Differ. 25 282-293 (2018)

42. Luedtke, D. A. et al. Inhibition of MCL1 enhances cell death induced by the Bcl-2-selective inhibitor ABT-199 in acute myeloid leukemia cells. Signal Transduct. Target Ther. 2, 17012 (2017).
43. Lin, K. H. et al. Targeting MCL1/BCL-XL forestalls the acquisition of resistance to ABT-199 in acute myeloid leukemia. Sci. Rep. 6, 27696 (2016).

44. Saenz, D. T. et al. BET protein bromodomain inhibitor-based combinations are highly active against postmyeloproliferative neoplasm secondary AML cells. Leukemia 31, 678-687 (2017).

45. Devarai, S. G. et al. HEXIM1 induction is mechanistically involved in mediating anti-AML activity of BET protein bromodomain antagonist. Leukemia $\mathbf{3 0}$, 504-508 (2016).

46. Bui, M. H. et al. preclinical characterization of BET family bromodomain inhibitor ABBV-075 suggests combination therapeutic strategies. Cancer Res. 77, 2976-2989 (2017).

47. Lin, $X$. et al. HEXIM1 as a robust pharmacodynamic marker for monitoring target engagement of BET family bromodomain inhibitors in tumors and surrogate tissues. Mol. Cancer Ther. 16, 388-396 (2017).

48. Roberts, A. W. et al. Targeting BCL2 with venetoclax in relapsed chronic lymphocytic leukemia. N. Engl. J. Med. 374, 311-322 (2016).

49. Fresquet, V., Rieger, M., Carolis, C., García-Barchino, M. J. \& Martinez-Climent, J. A. Acquired mutations in BCL2 family proteins conferring resistance to the BH3 mimetic ABT-199 in lymphoma. Blood 123, 4111-4119 (2014).

50. Rudin, C. M. et al. Phase II study of single-agent navitoclax (ABT-263) and biomarker correlates in patients with relapsed small cell lung cancer. Clin. Cancer Res. 18, 3163-3169 (2012).

51. Roberts, A. W. et al. Substantial susceptibility of chronic lymphocytic leukemia to BCL2 inhibition: results of a phase I study of navitoclax in patients with relapsed or refractory disease. J. Clin. Oncol. 30, 488-496 (2012).

52. Zhong, Q., Gao, W., Du, F. \& Wang, X. Mule/ARF-BP1, a BH3-only E3 ubiquitin ligase, catalyzes the polyubiquitination of MCL1 and regulates apoptosis. Cell 121, 1085-1095 (2005).

53. Schwickart, M. et al. Deubiquitinase USP9X stabilizes MCL1 and promotes tumour cell survival. Nature 463, 103-107 (2010).

54. Dey, J. et al. Voruciclib, a clinical stage oral CDK9 inhibitor, represses MCL1 and sensitizes high-risk Diffuse Large B-cell Lymphoma to BCL2 inhibition. Sci. Rep. 7, 18007 (2017)

55. Villunger, A. et al. p53- and drug-induced apoptotic responses mediated by BH3-only proteins puma and noxa. Science 302, 1036-1038 (2003).

56. Pan, R. et al. Synthetic lethality of combined Bcl-2 inhibition and p53 activation in AML: mechanisms and superior antileukemic efficacy. Cancer Cell 32 748-760 (2017)

57. Ruefli-Brasse, A. \& Reed, J. C. Therapeutics targeting BCl-2 in hematological malignancies. Biochem. J. 474, 3643-3657 (2017).

58. Kojima, $\mathrm{K}$. et al. Concomitant inhibition of MDM2 and BCl-2 protein function synergistically induce mitochondrial apoptosis in AML. Cell Cycle 5, 2778-2786 (2006).

59. Chan, S. M. et al. Isocitrate dehydrogenase 1 and 2 mutations induce BCL-2 dependence in acute myeloid leukemia. Nat. Med. 21, 178-184 (2015).

60. Lagadinou, E. D. et al. BCL-2 inhibition targets oxidative phosphorylation and selectively eradicates quiescent human leukemia stem cells. Cell Stem Cell 12 329-341 (2013). 